$$
\text { CONF-950684--2 }
$$

\title{
X-RAY DIFFRACTION CHARACTERIZATION OF THIN SUPERCONDUCTIVE FILMS
}

\author{
K. J. Kozaczek ${ }^{1}$, G. W. Book ${ }^{2}$, T. R. Watkins ${ }^{1}$, W. B. Carter ${ }^{2}$ \\ 'High Temperature Materials Laboratory, Oak Ridge National Laboratory, Oak Ridge. \\ TN 37831-6064, USA \\ ${ }^{2}$ School of Materials Science and Engineering, Georgia Institute of Technology, Atlanta. \\ GA 30332-0245, USA
}

Keywords: texture, thin films, high $T_{c}$ superconductors, $x$-ray diffraction.

\begin{abstract}
The physical and mechanical properties of thin films are often different from the properties of bulk material and are dictated by the film/substrate orientation relationship, crystal anisotropy and crystallographic texture of the film. X-ray diffraction texture analysis provides information about preferential film growth and can be used for optimization of deposition parameters and prediction of properties of thin films.

An $\mathrm{x}$-ray back reflection technique using the Bragg-Brentano geometry with experimental corrections for absorption and defocusing was used to study thin ceramic films deposited by combustion chemical vapor deposition (CCVD). The film/substrate orientation relationships of $\mathrm{YBa}_{2} \mathrm{Cu}_{3} \mathrm{O}_{\mathrm{x}}(\mathrm{YBCO})$ superconducting thin films deposited via $\mathrm{CCVD}$ on single crystal $\mathrm{MgO}$ and polycrystalline silver substrates were studied. The as-deposited films on single crystal (100) $\mathrm{MgO}$ substrates showed strong preferential growth with the basal plane parallel to the substrate surface (caxis up growth). Texture analysis showed two in-plane alignment orientations of the film with respect to the substrate, with YBCO [100] and [110] aligned with the [100] $\mathrm{MgO}$ substrate. YBCO films deposited on cold-rolled polycrystalline silver displayed $\mathrm{c}$-axis up growth indicating that the orientation of the polycrystalline substrate (brass type texture) did not induce detectable in-plane preferential growth of the YBCO.
\end{abstract}

\section{Introduction}

Thin layers with thickriesses from nanometers to hundreds of micrometers are of great interest in solid state physics and materials science. In recent years, new technologies for depositing thin, high $T_{c}$ superconductive films were developed for potential applications such as magnetic shielding, infra-red detectors and high-powered, light weight motors. Due to the anisotropy of most of the physical properties of single crystals and the fact that most of the film depositing techniques result in a preferred crystallographic orientation of the polycrystalline film. the electric. magnetic, and mechanical properties of thin films are anisotropic [1]. Furthermore, the surface roughness, rate of impurity diffusion and residual stress are correlated with the texture [2]. Quantitative texture analysis is a tool which provides the information necessary to control and optimize the manufacturing process and to predict specific physical properties. In recent years significant progress has been made in developing experimental techniques for nondestructive texture analysis in thin films. X-ray diffraction techniques such as $\Theta / 2 \Theta[3,4]$, rocking curve [4], and pole density [1,2] in the grazing incidence or BraggBrentano incidence are used to determine the orientation of single or multiple layers deposited on single crystal or polycrystalline substrates. Single pole or multiple pole density measurements combined with quantitative texture analysis [e.g., 5] emerged as powerful tools for characterization and prediction of properties of thin films. 
YBCO films were deposited on a (100) oriented single crystal of $\mathrm{MgO}$ and cold rolled polycrystalline silver by the gaseous fuel combustion chemical vapor deposition (CCVD) technique [21-23]. The substrates were placed with the surface normal at an angle of 35 degrees with respect to the flame direction, and the flame temperature in the vicinity of the surface was maintained at approximately $1170 \mathrm{~K}$. The film was deposited at the rate of approximately $1 \mu \mathrm{m} /$ hour to a final thickness of 0.5-1.0 $\mu \mathrm{m}$. Subsequently, samples were subjected to a two part annealing procedure. The samples were maintained at $1120 \mathrm{~K}$ for 1 hour under a $2 \%$ oxygen/ $98 \%$ nitrogen atmosphere. The temperature was then reduced to $760 \mathrm{~K}$ at a rate of $5 \mathrm{~K} / \mathrm{min}$ and held for two hours in $100 \%$ oxygen. Annealing was conducted at atmospheric pressure. The average in-plane grain size was approximately $10 \mu \mathrm{m}$. The studied samples reached zero resistance at $78 \mathrm{~K}$ with the transition onset beginning at $84 \mathrm{~K}$.

\section{X-ray diffraction characterization}

A four circle powder-texture-residual stress goniometer [24] was used for $x$-ray diffraction measurements. $\mathrm{CrK}_{\alpha}$ radiation (wavelength $\lambda=0.22897 \mathrm{~nm}$ ) from an $\mathrm{x}$-ray tube operating at $45 \mathrm{kV}$ and $40 \mathrm{~mA}$ was used for all $\mathrm{x}$-ray diffraction experiments. The $\mathrm{x}$-ray beam employed had a diameter and divergence of $2 \mathrm{~mm}$ and $0.2^{\circ}$, respectively. The diffracted $\mathrm{x}$-ray beam was registered by a solid state detector eliminating the $\mathrm{CrK}_{\beta}$ and continuous spectrum radiation; the use of monochromatic radiation is essential for thin films deposited on single crystal substrates in order to eliminate spurious peaks [25]. Due to the sharp texture, the relatively large grain size of the film. and the small $\mathrm{MgO}$ sample size an incident beam with a high divergence (no divergence slits) and the projection on the sample plane larger than the sample itself was used. Thus the diffracting volume varied for different sample tilts. The penetration depth $t_{99 \%}$ for all sample tilts exceeded the film thickness $(1.5 \mu \mathrm{m}$ to 10 $\mu \mathrm{m}$ for the linear absorption coefficient $\mu=3117 \mathrm{~cm}^{-1}$ and theoretical density for a fully dense material $\left.p=6.368 \mathrm{~g} / \mathrm{cm}^{3}\right)$. This allowed the collection of the (111), (200), and (220) pole figures from the $\mathrm{MgO}$ substrate through the film and the determination of the epitaxial relationships between the film and the substrate. The $\mathrm{x}$-ray intensities from the substrate were not corrected for absorption in the film since for single crystals (with strong and sharp reflections) the effect of absorption in the film layer is negligible and does not hinder quantitative analysis of texture in the substrate. Since the textured $\mathrm{Ag}$ substrate gives much weaker reflections, (111), (200), and (220) pole figures were collected from the uncoated side of the sample. Standard diffraction patterns from the films were collected in the range $2 \Theta=0-110^{\circ}$. Based on the diffraction patterns, several non-overlapping reflections were used for the pole figure measurements. The (112), (113), (006), (202), and (122) pole figures were collected for the film deposited on the silver substrate and (116)+(123), (101), (213), and (006) for the film on the $\mathrm{MgO}$ substrate. The pole figures were collected in the Schulz back reflection mode [7] with a continuous $\phi$ scan at a rate of $60 \% \mathrm{~min}$ and $\chi$ scan with a $5^{\circ}$ step, and corrected for background. The defocusing and absorption corrections were done experimentally using randomly oriented powder deposited on an amorphous substrate of the same size as the $\mathrm{MgO}$ and $\mathrm{Ag}$ substrates. Quantitative texture analysis was carried out using the popLA code [5].

\section{$\underline{\text { Results }}$}

The X-ray diffraction patterns for YBCO deposited by the CCVD technique on single crystal MgO and rolled polycrystalline silver are presented in Fig. 1 (a) and (b) respectively. The composition of the films is predominantly the $\mathrm{YBa}_{2} \mathrm{Cu}_{3} \mathrm{O}_{7}-\delta$ phase with $\mathrm{BaCuO}_{2}, \mathrm{CuO}$, and $\mathrm{YBaCuO}$ as minor phases. The YBCO peaks from the film deposited on $\mathrm{MgO}$ were fit individually and the lattice parameters were refined using a least-squares interpolation [26]. The calculated lattice parameters for the YBCO film were $a=0.3835 \mathrm{~nm}, b=0.3889 \mathrm{~nm}$, and $c=1.1687 \mathrm{~nm}$ and were used for the harmonic analysis of the pole figures. The multiple pole figure (116)+(123) was separated, and the $\mathrm{MgO}$ reflections were removed from the (213) pole figure prior to harmonic analysis. The harmonic expansion coefficients were calculated for the orthorhombic crystal symmetry, and the complete pole figures extrapolated.

Some of the complete pole figures for the film deposited on the $\mathrm{MgO}$ substrate are presented in Fig. 2. The multiple pole figure $(116)+(123)$ shows the $(116)$ poles at $\chi=35.5^{\circ}$, and (123) poles at $\chi=66^{\circ}$. The eight-fold symmetry of (116), (123), and (231) pole figures shows that the c-axis is perpendicular to the sample surface with a total angular spread of approximately $10^{\circ}$, with the [100] 


\section{X-ray diffraction texture measurements}

The standard pole density measurements employ symmetric Bragg-Brentano focusing geometry [6] in the Schulz back-reflection mode [7]. In this technique, the intensities of the diffracted $x$-rays from selected sets of crystallographic planes are registered covering the orientation sphere with the grid of $5^{\circ} \times 5^{\circ}$ in pole distance $\{\chi\}$ and azimuth $\{\phi\}$. The measured intensities have to be corrected for absorption and defocusing with increasing specimen tilt $\{\chi\}$. The intensity is proportional to the number of lattice planes that are suitably oriented for diffraction and, therefore. the diffracting volume should be kept constant during the measurements in order to extract information about the texture. The irradiated volume is a function of the irradiated area on the sample surface (the projection of the incident, collimated $x$-ray beam on the sample surface) and the effective depth of penetration. The effective depth of penetration depends on the sample linear absorption coefficient and the inclination angle(s) of the incident $x$-ray beam [7-9]. In the back-reflection technique, the effective penetration depth $t_{99 \%}$ is defined as the thickness of the near-surface layer in which the intensity of the incident beam is reduced $99 \%$ due to absorption. Each $\{\chi\}$ angle of the pole figure thus corresponds to another depth-average of the near-surface layer. This fact does not hinder the interpretation of texture in the case of thick (thicker than $t_{g g_{0}}$ ) samples with a homogeneous texture. In this case the irradiated surface area and the effective penetration depth change due to sample tilts in such a way that the irradiated volume remains constant for all tilts [7]. However, for thin surface layers the $x$-rays penetrate through the whole layer and the increase in irradiated area at higher $\chi$ tilts is not counter-balanced by the decrease in penetration depth causing the change of the irradiated volume during the sample tilting. Another source of the diffracted intensity variation is the so called defocusing effect. At large tilt angles, due to poor focusing conditions, not all the diffracted $x$-rays reach the detector and the registered intensity decreases.

Numerous theoretical corrections for the absorption and defocusing for finite thickness layers have been proposed $[2,3,7,10]$. An alternative approach is to derive the correction factor experimentally from a randomly oriented powder of finite thickness and under exactly the same experimental conditions as the actual measurements. The experimental correction is advantageous in that it eliminates all errors due to imperfect experimental conditions (e.g.. wobble in the sample rotation mechanism, poor incident and diffracted beam collimation) [11].

The fact that the sampling depth can be controlled by changing the incident angle of the $x$-ray beam has been used to measure textures in multilayers. layers with a texture gradient, and to study film/matrix orientation relationships $[3,9-14]$. The application of grazing incidence angle for pole figure measurements allows one to analyze textures in films as thin as tens of nanometers [9, 12].

\section{Texture study of the $\mathrm{YBa}_{2} \mathrm{Cu}_{3} \mathrm{O}_{\mathrm{x}}(\mathrm{YBCO})$ superconducting thin films}

$\mathrm{YBa}_{2} \mathrm{Cu}_{3} \mathrm{O} 7(\mathrm{YBCO})$ is a high $\mathrm{T}_{\mathrm{c}}$ compound which has an $\mathrm{D}_{2 \mathrm{~h}}^{1}$ orthorhombic unit cell (Pmmm, $\mathrm{D}^{1}{ }_{2 \mathrm{~h}}$. oP13, no. 47) with lattice parameters for the nominal composition $a=0.3825 \mathrm{~nm}, \mathrm{~b}=0.3886 \mathrm{~nm}$, and $\mathrm{c}=1.1660 \mathrm{~nm}$ [15]. The elastic properties of YBCO are moderately aniostropic [16] with the bulk $(B)$ and shear $(G)$ moduli estimated by Voight $(V)$ and Reuss $(R)$ averaging schemes as $B_{v}=116$, $\mathrm{B}_{\mathrm{R}}=113, \mathrm{G}_{\mathrm{V}}=63.7, \mathrm{G}_{\mathrm{R}}=54.4 \mathrm{GPa}$. The superconducting property in YBCO superconductors is restricted to the (001) crystallographic plane, and therefore a strong preferred orientation in a polycrystalline film is crucial in order to achive high current density. Because the critical current density along the c-axis is several orders of magnitude smaller than that parallel to the $\mathbf{a - b}$ plane $[17,18]$, a preferred orientation with the c-axis perpendicular to the substrate surface $\left(c_{1}\right)$ is desired. The critical current density, $J_{c}$, is also greatly affected by in-plane misoriented crystallites; grain boundary misorientation angles greater than $10^{\circ}$ cause a decrease in $\mathrm{J}_{\mathrm{c}}$ by two orders of magnitude [18$20]$.

The present study is concerned with the preferred orientation of thin YBCO films deposited on $\mathrm{MgO}$ single crystal and on polycrystalline cold-rolled substrates. Single crystal $\mathrm{MgO}$ is a commonly used substrate in the high temperature superconductor applications because it is chemically compatible with YBCO, exhibits a relatively low dielectric constant and is relatively inexpensive. Rolled silver substrates represent an attractive material for a tape due to good plasticity, electric and thermal conductivity, and chemical compatibility with superconductive films. 
and [110] directions parallel to the [100] $\mathrm{MgO}$. These preferred orientations are usually denoted as $\mathbf{c}_{10}$ and $\boldsymbol{c}_{145}$ textures, respectively. In addition to the epitaxial growth orientation, (001) in-plane misorientation with an angle of $45^{\circ}$ is one of the highly preferred misorientations resulting from a near coincidence site lattice (NCSL) formation between YBCO and $\mathrm{MgO}$ [20]. The volumetric fractions of $\mathbf{c}_{10}$ and $\mathbf{c}_{145}$ texture components were estimated by integrating the intensities of the (116) reflection corresponding to these two orientations. The estimated volume fractions are $67 \%$ of $\mathbf{c}_{10}$ and $33 \%$ of $\mathbf{c}_{145}$ textures. The existence of the two distinct groups of orientations implies that there is a large fraction of high angle grain boundaries (misorientation close to $45^{\circ}$ around the c-axis). The degree of elastic anisotropy in YBCO was estimated using the law of mixtures along with the elastic constants of YBCO single crystals [16] and volumetric tractions of textures. The calculated elastic moduli were $\mathrm{E}_{0 \dot{0} \mathrm{l}}=76.9 \mathrm{GPa}, \mathrm{E}_{1 \dot{\omega} \mathrm{w}}=126 \mathrm{GPa}$, and $\mathrm{E}_{110}=83.6 \mathrm{GPa}$ (where the subscript 001 denotes the direction perpendicular to film surface, or c-axis direction, and 100 and 110 denote the directions parallel to (100) $\mathrm{MgO}$ and $45^{\circ}$ with respect to [100] $\mathrm{MgO}$, respectively) which show a substantial in-plane elastic anisotropy.

Fig. 3 shows inverse pole figures for the silver substrate obtained from the WMV analysis [5] of the experimental pole figures. The silver substrate had a moderate $(110)<112>$ type texture typical for rolled low stacking fault energy metals (e.g., [1, 27]). Some of the experimental pole figures for YBCO deposited on silver are shown in Fig. 4. The YBCO film displayed a very strong and sharp wire $c_{\perp}$-type texture with the angular spread around the substrate surface normal of less than 5 degrees. The (113) pole figure shows a relatively uniform distribution of the a-axes in the plane of the substrate. Hence, there is no epitaxial relationship between the film and the substrate. The wire $c_{1}-t y p e$ texture provides for a continuous distribution of the in-plane grain boundary misorientation angles and uniform in-plane elastic properties (E=104 GPa).

\section{Summary}

An x-ray back reflection technique using the Bragg-Brentano geometry with experimental corrections for absorption and defocusing was used to study thin ceramic films deposited by combustion chemical vapor deposition (CCVD). The film/substrate orientation relationships of $\mathrm{YBa}_{2} \mathrm{Cu}_{3} \mathrm{O}_{\mathrm{X}}$ (YBCO) superconducting thin films deposited via $\mathrm{CCVD}$ on single crystal $\mathrm{MgO}$ and polycrystalline silver substrates were studied. The as-deposited films on single crystal (100) $\mathrm{MgO}$ substrates showed strongly preferential growth with the basal plane parallel to the substrate surface (caxis up growth). Texture analysis showed two in-plane alignment orientations of the film with respect to the substrate, with YBCO [100] and [110] aligned with the [100] $\mathrm{MgO}$ substrate. YBCO films deposited on roll-textured polycrystalline silver displayed c-axis up growth indicating that the orientation of the polycrystalline substrate did not induce detectable in-plane preferential growth of the YBCO.

\section{Acknowledgments}

The authors thank Dr. S.T. Misture for the lattice parameter refinement analysis. This project was sponsored by the U. S. Department of Energy. Assistant Secretary for Energy Efficiency and Renewable Energy. Office of Transportation Technologies, as part of the High Temperature Materials Laboratory User Program under contract DE-AC05-84OR2,1400, managed by Marin Marietta Energy Systems. Inc.

\section{DISCLAIMER}

\footnotetext{
This report was prepared as an account of work sponsored by an agency of the United States Government. Neither the United States Government nor any agency thereof, nor any of their employees, makes any warranty, express or implied, or assumes any legal liability or responsibility for the accuracy, completeness, or usefulness of any information, apparatus, product, or process disclosed, or represents that its use would not infringe privately owned rights. Reference herein to any specific commercial product, process, or service by trade name, trademark, manufacturer, or otherwise does not necessarily constitute or imply its endorsement, recommendation, or favoring by the United States Government or any agency thereof. The views and opinions of authors expressed herein do not necessarily state or reflect those of the United States Government or any agency thereof.
} 
[1] H.-J. Bunge, Texture Analysis in Materials Science (Butterworths, London, 1982).

[2] H.R. Wenk, M. Sintubin, J. Huang, G.C. Johnson, R.T. Howe, "Texture analysis of polycrystalline silicon films," J. Appl. Phys. 67 (1), 1 (1990).

[3] T.I. Kamins, Polycrystalline Silicon for Integrated Circut Applications (Kluwer Academic, Boston, 1988).

[4] A. Tizlioune, J. Bessieres, J.J. Heizmann, and J.F. Bobo, "Texture in aluminum and iron thin films and texture of bilayers Al/Fe deposited on silicon," Materials Science Forum Vol. 157-162, 1487 (1994).

[5] J.S. Kallend, U.F. Kocks, A.D. Rollett, and H.R. Wenk, popLA-Preferred Orientation PackageLos Alamos (Los Alamos National Laboratory, Los Alamos, 1993).

[6] B.D. Cullity, Elements of X-Ray Diffiraction (Addison Weseley, Reading, MA, 1978).

[7] L.G. Schulz, J. Appl. Phys. 20, 1033 (1949).

[8] J.T. Bonarski, L. Wcisak, and H.J. Bunge, "Investigaiton of inhomogeneous textures of coatings and near-surface-layers," Materials Science Forum Vol. 157-162, 111 (1994).

[9] B. Moreau, F. Wagner, and H. Goebel, "Optimization of the texture determination of thin films from X-ray diffraction measurements," Materials Science Forum Vol. 157-162, 159 (1994).

[10] D. Chateigner, P. Germi, and M. Pernet, "Texture analysis by the Schulz reflection method: defocalization corrections for thin films," J. Appl. Cryst. 25, 766 (1992).

[11] J. A. Szpunar and P. Blandford, "Equipment for texture measurement in thin films," Materials Science Forum Vol. 157-162, 207 (1994).

[12] G. Berming, J. Tobisch, and K. Helming "Quantitative texture analysis of thin polycrystalline layers," Materials Science Forum Vol. 157-162, 97 (1994).

[13] D. Chateigner, P. Germi, and M. Pernet, "X-ray texture analysis in films by the reflection method: principal aspects and applications," Materials Science Forum Vol. 157-162, 1379 (1994).

[14] D. Chateigner, P. Germi, and M. Pernet, "X-ray texture analysis of thin films by the reflection method: intermediate regime in defocusing conrections," J. Appl. Cryst. 27, 278 (1994).

[15] A.Reller, J. Bednorz, K. Muller, Z. Phys. B: Condens. Matter Quanta 67, 285 (1987).

[16] H. Ledbetter and M. Lei, "Monocrystal elastic constants of orthotropic YBa2Cu3O7: An estimate," J. Mater. Res. 11 (11), 2253 (Nov. 1991).

[17] D. Dimos, P. Chauhari, C.C. Tsuei, and T.R. McGuire, Phys. Rev. Lett. 61, 2476 (1988).

[18] T. R. Dinger, T.K. Worthington, W.V. Gallagher, and R.L. Saddstrom, Phys. Rev. Lett. 58, 2687 (1987).

[19] M.F. Chisholm and S.J. Peenycook, "Structural origin of reduced critical currents at $\mathrm{YBa}_{2} \mathrm{Cu}_{3} \mathrm{O}_{7-\delta} \delta$ grain boundaries," Nature, 351, 47 (1991).

[20] H. Suzuki, Y. Fujiwara, Y. Hirotsu, J. Chen, K. Nakajima, and T. Yamashita, "Natural and artificial grain boundaries of YBCO thin films for Josephson junctions," Physica C, 190, 75 (1991).

[21] G.W. Book, W.B. Carter, T.A. Polley, and K.J. Kozaczek, "Preparation of $\mathrm{YBa}_{2} \mathrm{Cu}_{3} \mathrm{O}_{\mathrm{X}}$ superconducting thin films via combustion chemichal vapor deposition," submitted to Thin Solid Films.

[22] W. B. Carter, W.W. Book, and A.T. Hunt, International Conference on Novel Techniques in Synthesis and Processing of Advanced Materials, TMS/ASM Materials Week Conference, Rosemont, Illinois, October 2-6, (1994) in press.

[23] A.T. Hunt, W.B. Carter, and J.K. Cochran, Jr., Phys. Lett., 63 (2), 266 (1993).

[24] H. Krause, A. Haase, "X-ray diffraction system PTS for powder, texture and stress analysis," Experimental Techniques of Texture Analysis, ed. H.J. Bunge, 405 (DGM, Oberusel, 1986).

[25] H.R. Wenk, "Advantages of monochromatic X-rays for Texture Determination of Superconducting Thin Films," J. Appl. Cryst., 25, 524 (1992).

[26] C.R. Hubbard, S.M. Lederman, and N.P. Pyrros, JCPDS-NBS*LSQ82, U.S. National Bureau of Standards (1982).

[27] U. Schmidt and K. Lucke, "Recrystallization textures of silver, copper and $\alpha$-brasses with different zinc-contents as a function of the rolling temperature," Texture, 3 (2), 85 (1979). 


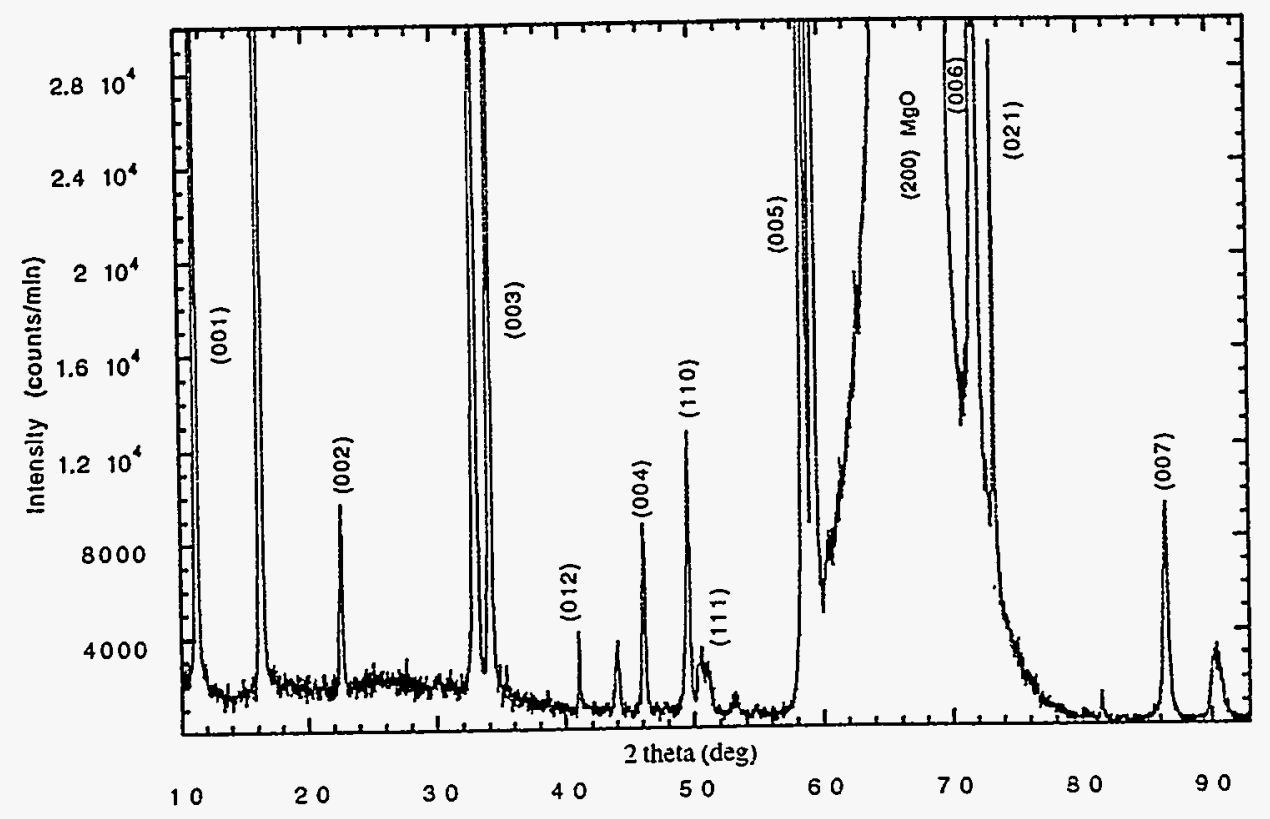

(a)

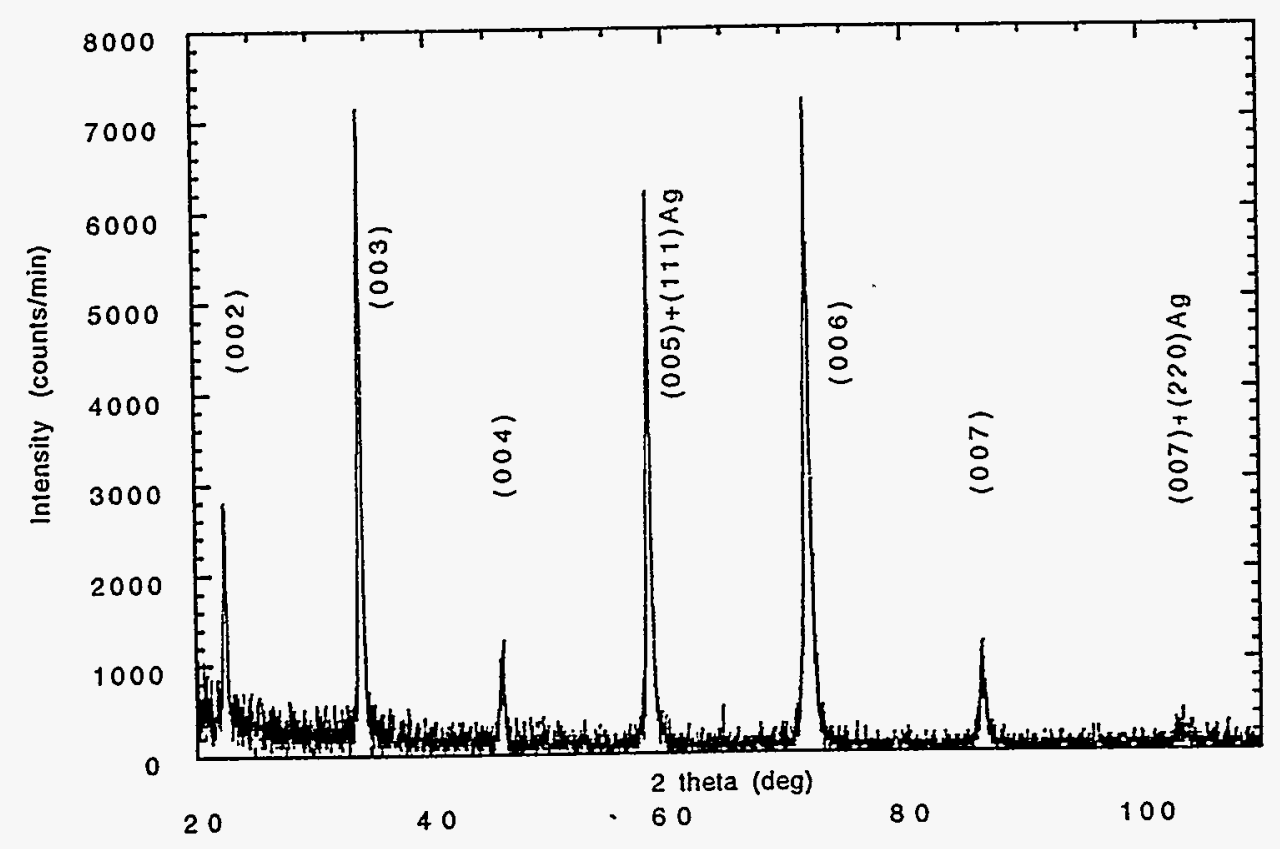

(b)

Figure 1. Diffraction patterns for YBCO films deposited on single crystal MgO (a), and cold-rolled silver (b). 

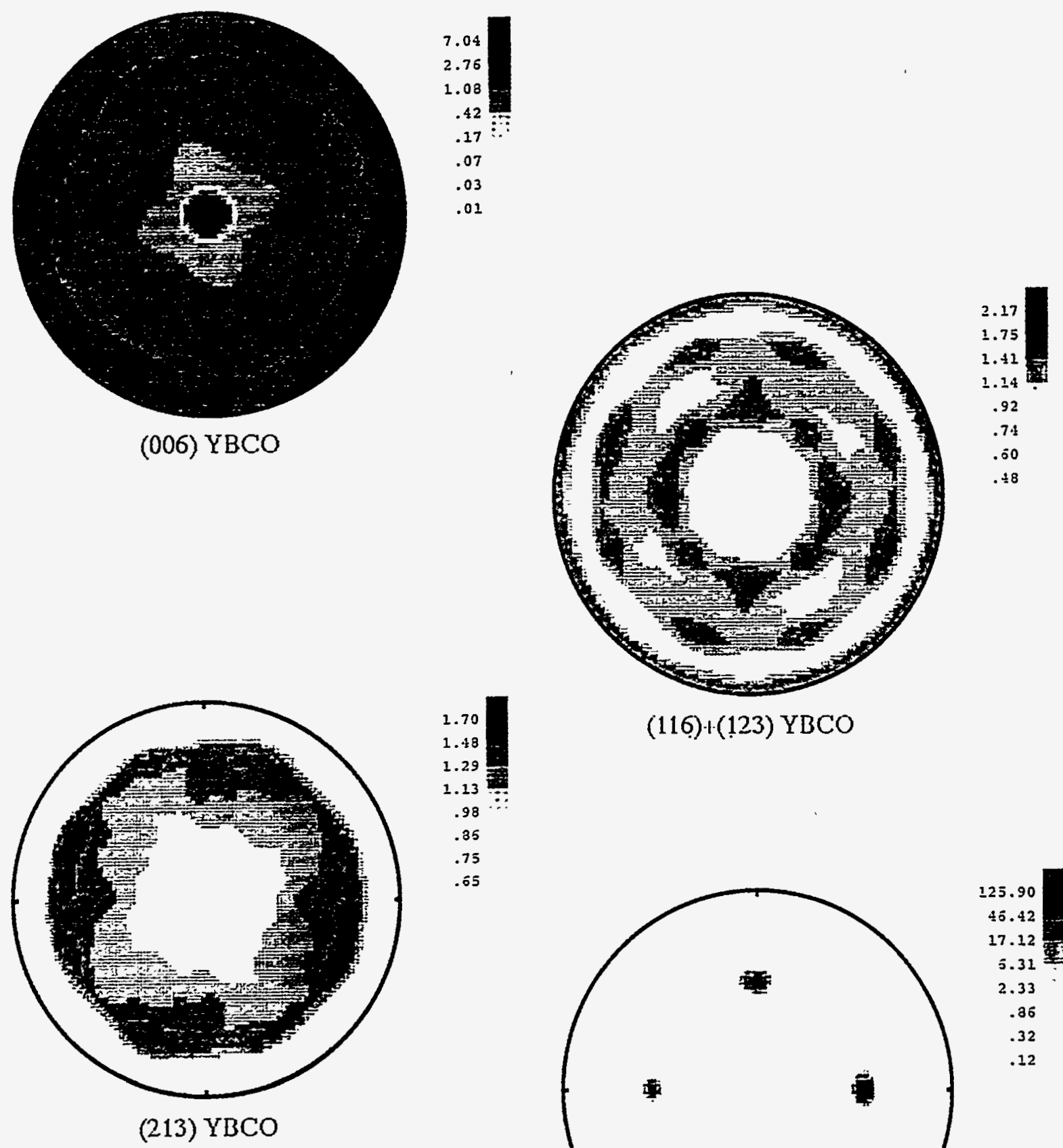

(116)+(123) YBCO

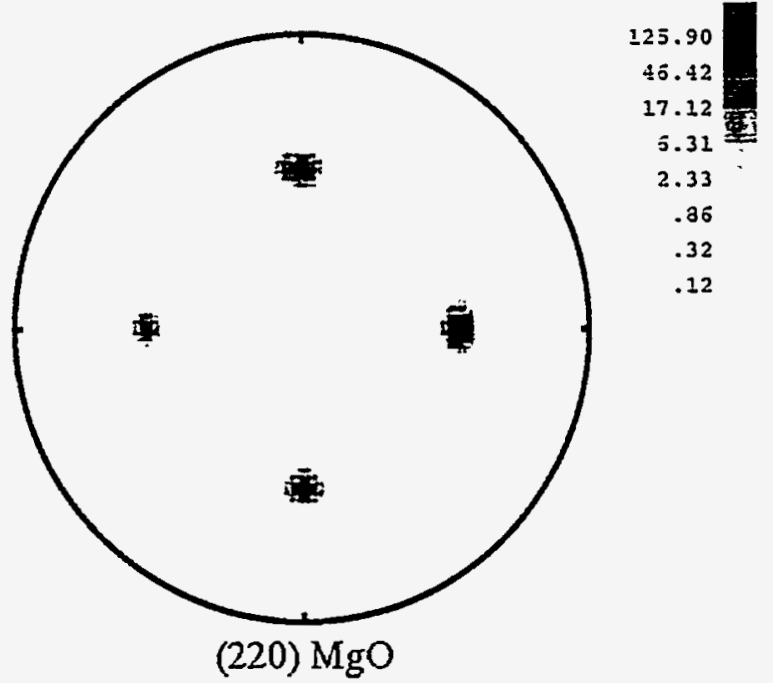

Figure 2. Pole figures for YBCO film and MgO substrate (stereographic projection, logarithmic intensity scale). 


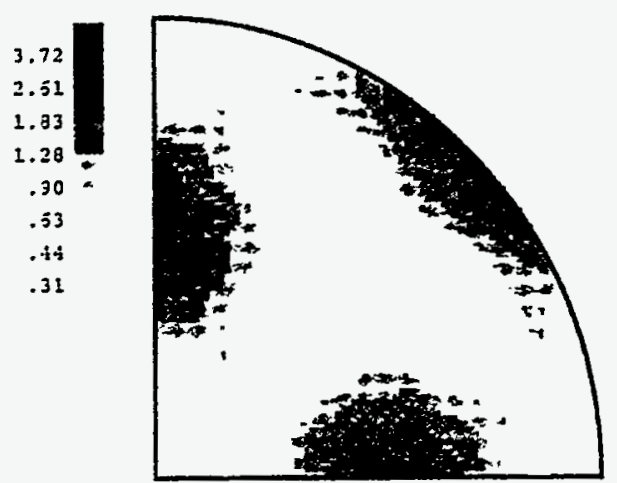

(a)
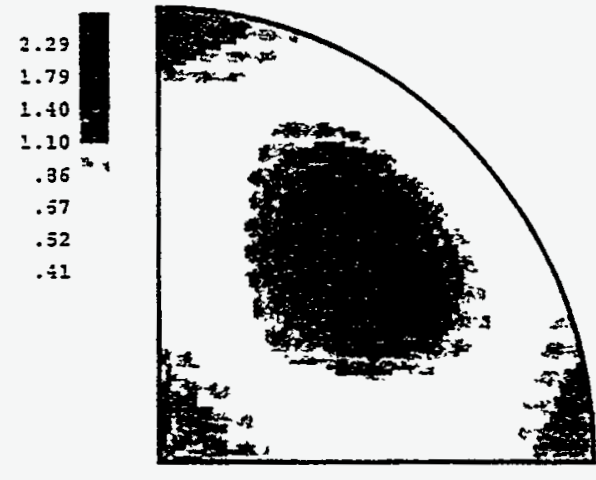

(b)

Figure 3. Inverse pole figures for silver substrate (a) surface normal (b) rolling direction. Equal area projection. logarithmic intensity scale.
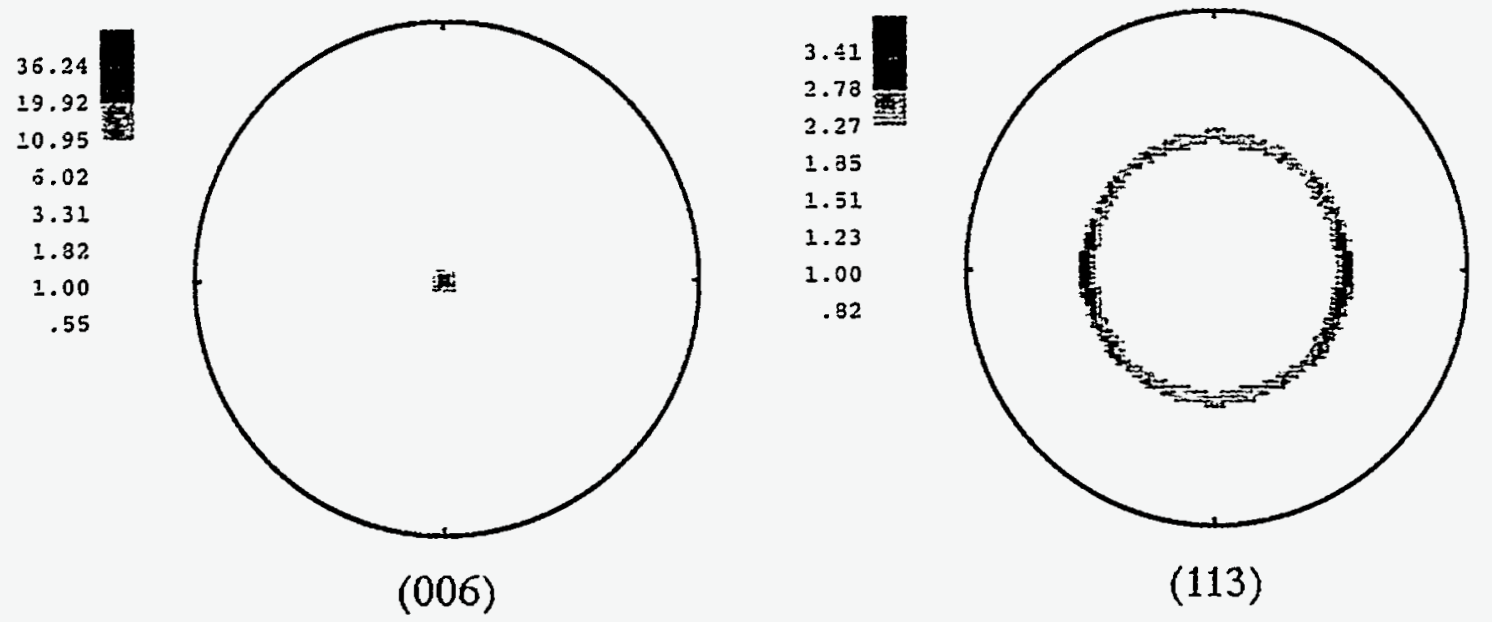

Figure 4. Experimental pole figures for YBCO film deposited on silver substrate (stereographic projection. logarithmic intensity scale). 